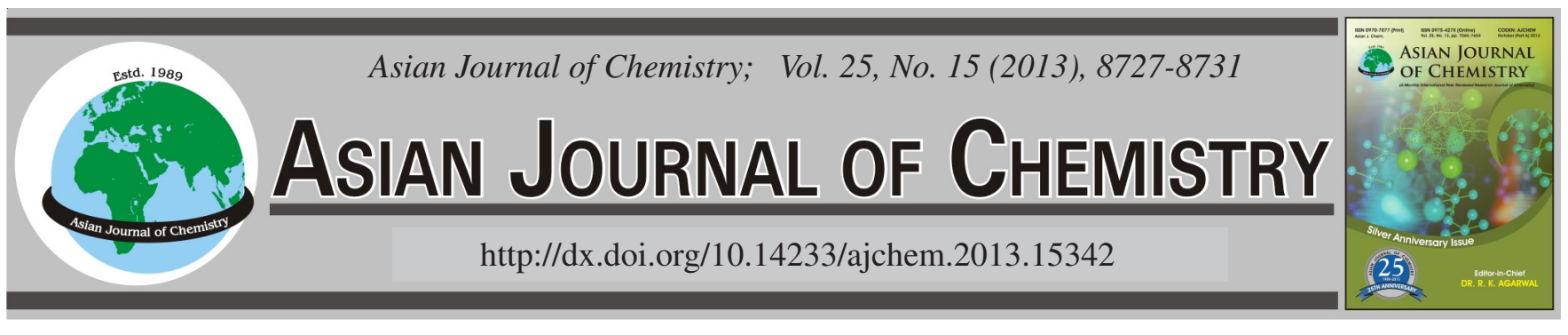

\title{
Systemic Delivery of Allium cepa L. var agrogatum Don Flavonoid-Rich Extract via the Percutaneous Route Using a Microemulsion System
}

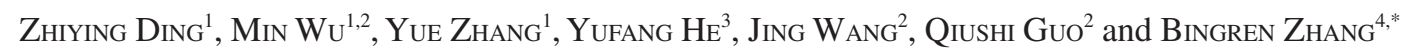

\author{
${ }^{1}$ School of Pharmaceutical Sciences, Jilin University, Changchun 130021, P.R. China \\ ${ }^{2}$ First Hospital, Jilin University, Changchun 130021, P.R. China \\ ${ }^{3}$ Jilin Academy of Chinese Medicine Sciences, Changchun 130012 , P.R. China \\ ${ }^{4}$ College of Instrumentation and Electrical Engineering, Jilin University, 938 Ximinzhu Street, Chaoyang District, Changchun City, Jilin Province, \\ P.R. China
}

*Corresponding author: Tel: +86 13843110698; E-mail: zbr@jlu.edu.cn

Key Words: Allium cepa L. var agrogatum Don flavonoid-rich extract, Microemulsion, Percutaneous absorption, Transcellular.

\section{INTRODUCTION}

Allium cepa L.var. agrogatum Don (ACAD) is a perennial flavonoid-rich food of the Liliaceae family native to northern China. The Allium cepa L. var agrogatum Don flavonoid-rich extract (ACADFE) contains compounds of the group of quercetin, which is a characteristic of members of the Liliaceae family ${ }^{1}$. Quercetin (QT) i.e., quercetin-4'-O- $\beta$-D-glucopyranoside and quercetin-3,4'-O- $\beta$-D-glucopyranoside are the predominant components of ACADFE, with contents of 157.9 $\pm 23.1,181.7$ \pm 25.4 and $199.3 \pm 27.8 \mathrm{mg} / \mathrm{g}$, respectively. ACADFE exhibits multiple biological activities, including antihyperlipidemic activity $^{2,3}$, blood fat regulatory activity, hemangiectasic activity ${ }^{4}$ and anti-atherosclerosis activity ${ }^{5,6}$ in addition, ACADFE acts as an antioxidant, removing free radicals and preventing oxidative damage $\mathrm{e}^{7-9}$. To date, a pharmaceutical dosage of ACADFE has not been reported. The quercetin in ACADFE is only sparingly soluble in water, which may be responsible for the limited absorption of quercetin in vivo. This limited absorption impairs the development of a topical pharmaceutical form of ACADFE; therefore, an alternative mode of delivery is desirable. In this investigation, an attempt has been made to transdermally deliver ACADFE across the skin using microemulsions.

A microemulsion is defined as an o/w or w/o emulsion that is transparent and has a droplet size from $10-100 \mathrm{~nm}^{10}$. Microemulsions are defined as "a system of water, oil and one or more amphiphilic molecules which is a single optically isotropic and thermodynamically stable liquid solution" Microemulsions have been widely used to improve the solubility and bioavailability of a number of poorly soluble drugs ${ }^{12}$. Microemulsions are useful as vehicles for transdermal drug delivery ${ }^{13-15}$.

Transdermal drug delivery is a convenient method of drug administration that enables physicians to provide controlled delivery of drugs to patients with minimal discomfort ${ }^{16}$. Transdermal drug delivery offers many benefits over delivery via the oral ${ }^{17,18}$, intravenous and injection routes ${ }^{19,20}$, which often result in adverse clinical side effects and/or fail to deliver the active ingredient to the intended organ either fast enough or in sufficiently high concentrations to treat a variety of systemic diseases $^{14}$.

In this paper, we report a new microemulsion formulation used for the transdermal delivery of ACADFE. We formulated 
a non-alcoholic microemulsion containing ACADFE and 'generally recognised as safe' inactive ingredients. The transdermal application of the ACADFE microemulsion was studied in hairless rats and the routes of skin permeability were determined through fluorescence images.

\section{EXPERIMENTAL}

Allium cepa L. var agrogatum Don flavonoid-rich extract (ACADFE) was purchased from the Department of Pharmaceutical Chemistry, School of Pharmaceutical Sciences, JiLin University (Changchun 130012, PR China). Polyethylene glycol 400 (PEG 400) and isopropyl myristate (IPM) were purchased from Tianjin Guangfujingxi Chemical Research Institute (Tianjin, China). Tween ${ }^{\circledR} 80$ and $\operatorname{Span}^{\circledR} 80$ were purchased from Tianjin Fuchen Chemical Reagent Factory (Tianjin, China). Water was purified by double distillation in a glass apparatus. All other chemical and solvents were analytical grade.

Preparation of the microemulsion: Generally, microemulsions were prepared by mixing surfactants (Tween ${ }^{\circledR} 80$, Span ${ }^{\circledR} 80$ ) and oil (isopropyl myristate) with a co-surfactant $(\text { PEG } 400)^{21}$. The appropriate volumes of water were added and ACADFE was then added to the prepared microemulsion ${ }^{22}$. The monophasic formulations were prepared spontaneously at room temperature. The co-surfactant-surfactant $(\mathrm{CoS} / \mathrm{S})$ weight ratio was $1: 1$ and the oil-Smix $(\mathrm{CoS}+\mathrm{S})$ weigh ratio was $3: 7$.

Construction of phase diagrams: Pseudo-ternary phase diagrams were constructed using the water titration method ${ }^{23}$. Pseudo-ternary phase diagrams of oil, distilled water and cosurfactant (CoS)-surfactant (S) mixtures were constructed at fixed $\mathrm{CoS} / \mathrm{S}$ weight ratios $^{24}$. The weight ratio of the two surfactants, Tween ${ }^{\circledR} 80$ and $\operatorname{Span}^{\circledR} 80$, was fixed and kept constant. The phase diagrams were obtained by visual inspection of mixtures of the ingredients, which were pre-weighed into glass vials, titrated with water and stirred well at room temperature. Because of the convenience of the method, the construction of the phase diagrams was performed by drawing 'water dilution lines' representing an increase in the water content and a decrease in the CoS-S and oil levels. The water was titrated along the dilution lines drawn from the water apex to the opposite oil side of the triangle. When the solution became turbid and a phase separation occurred, the samples were considered as biphasic. Mixtures that were clear and transparent after stirring were considered monophasic and these samples were marked as points in the phase diagram. The area covered by these points was considered to be the region in which the oil in water $(\mathrm{o} / \mathrm{w})$ microemulsion existed.

The formulation were $\operatorname{Span}^{\circledR} 80$ and Tween ${ }^{\circledR} 80$ at a 1:2.5 w/w ratio and PEG 400 at a co-surfactant/surfactant $(\mathrm{CoS} / \mathrm{S})$ ratio of 1:1. Upon gentle shaking of the mixture, a clear liquid spontaneously formed with no need for special agitation/ equipment.

\section{Microemulsion characterization}

Dynamic light scattering: The average droplet size and the zeta potential of the microemulsions were measured by dynamic light scattering using a Zetasizer Nano-ZS (Malvern Instruments, England) at $25^{\circ} \mathrm{C}^{21}$.
Stability of microemulsions: The stability study was conducted for the microemulsion formulation. Samples of sufficient size were placed in amber-coloured containers and were placed at $4 \pm 2{ }^{\circ} \mathrm{C} / 75 \pm 5 \%$ R.H., $25 \pm 2{ }^{\circ} \mathrm{C} / 75 \pm 5 \%$ R.H. and $40 \pm 2{ }^{\circ} \mathrm{C} / 75 \pm 5 \%$ R.H. for 3 months. Aliquots were withdrawn at time intervals of 1,2 and 3 months ${ }^{25}$. The stability was investi-gated by monitoring the samples for the occurrence of a dispersed phase or crystals after centrifugation. The drug content in the formulations after centrifugation was estimated using HPLC.

in Vivo studies-animals: The rats (7-10 days old, Wister, from the Faculty of Experimental Animals of Jilin University, Changchun, PR China) were housed individually with free access to food and water. Experimental protocols for the animals used in this study were reviewed by the Animal Care and Use Committee of the College of Pharmacy. A $12 \mathrm{~h}$ light/ $12 \mathrm{~h}$ dark cycle was used to maintain the normal circadian rhythm of the animals.

in Vivo targeting studies via the percutaneous route: On the day of the experiment, $2 \mathrm{~mL}$ of the microemulsion formulation was applied to a limited area $\left(2 \mathrm{~cm}^{2}\right)$ on the dorsal skin of each hairless rat. At specific time points up to $12 \mathrm{~h}$, the animals were killed and the heart, liver, spleen, lungs and kidneys were carefully excised ${ }^{26,27}$. These tissues were washed with the fresh distilled water, wiped with a cotton swab and weighed. Subsequently, all tissues were homogenised. Then, $1 \mathrm{~mL}$ of methanol was added and the mixture was centrifuged at $12000 \mathrm{rpm}$ for $15 \mathrm{~min}^{28,29}$. The supernatant was filtered through a $0.22 \mu \mathrm{m}$ pore size cellulose membrane filter and the filtrate was analyzed by HPLC, as described previously. Each data point represented the average of three determinations.

HPLC analysis: The concentrations of the sample in the tissues were determined by UV-HPLC analysis using a Shimadzu (Kyoto, Japan) liquid chromatograph equipped with an LC-10 AT VP solvent pump unit and an SPD-10A VP UVvisible detector. The chromatographic column was a Kromasil $\mathrm{C}_{18}$ column $(5 \mu \mathrm{m}, 250 \mathrm{~mm} \times 4.6 \mathrm{~mm})$. A mixture of methanol and $0.4 \%$ phosphoric acid solution (51:49) was used as the mobile phase. The flow rate was $1.0 \mathrm{~mL} / \mathrm{min}$, the injection volume was $10 \mu \mathrm{L}$, UV-detection was performed at a wavelength of $365 \mathrm{~nm}$ and the retention time was $8.9 \mathrm{~min}$. No interface from the other formulation components was observed. All samples were filtered through a $0.2 \mu \mathrm{m}$ pore size membrane filter $\left(0.2 \mu \mathrm{m}\right.$ Nylon, Millipore Millex-GN) before injection ${ }^{11}$. The peak area correlated linearly with concentration in the range of $0.268-85.816 \mu \mathrm{g} \mathrm{mL} \mathrm{m}^{-1}, \mathrm{r}=0.9998$.

in Vivo skin permeation studies using confocal laser scanning microscopy (CLSM): On the day of the experiment, $2 \mathrm{~mL}$ of the microemulsion formulation was applied to a limited area $\left(2 \mathrm{~cm}^{2}\right)$ on the dorsal skin of each rat. The formulations were left there for $10 \mathrm{~min}$ or $7 \mathrm{~h}$ to allow the penetration of ACADFE into the skin and its conversion into a fluorescent molecule. At the end of the experiment, the rats were sacrificed. The formulation-exposed skin areas were removed from the animals for confocal scanning laser microscopy analysis. These skin samples were frozen in liquid nitrogen. Cryosections were prepared perpendicular to the skin's surface using a cryomicrotome (Microm D-6900, Germany). The skin slices were 

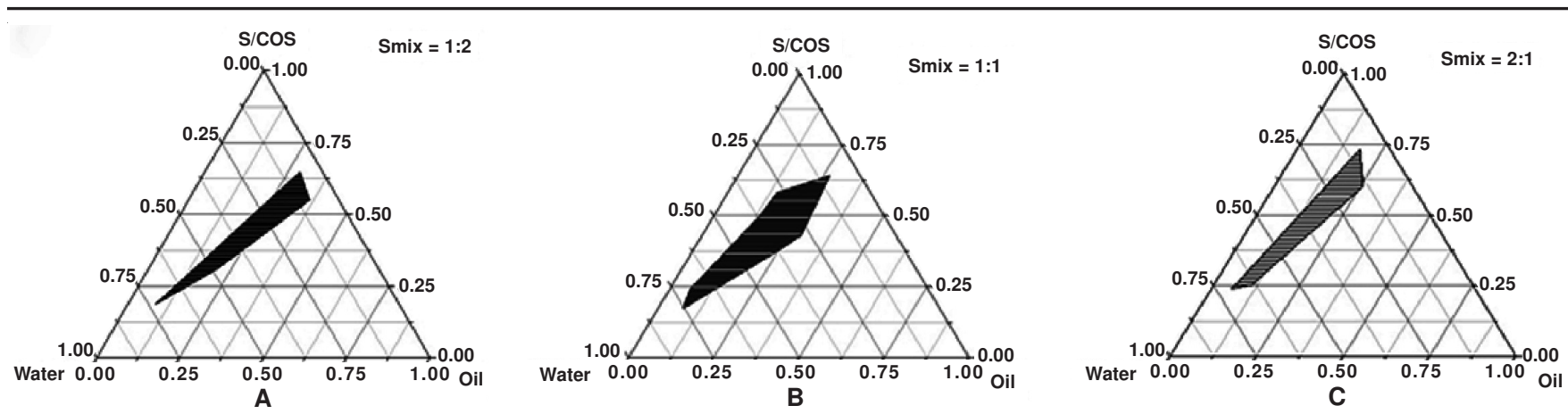

Fig. 1. Pseudo-ternary phase diagrams of microemulsions consisting of Tween 80 and PEG 400 as the surfactant and cosurfactant, respectively, at ratios of (A) 1:2 (w/w), (B) 1:1 (w/w) and (C) 2:1 (w/w). The surfactant was composed of Tween 80 and Span 80 at a ratio of 1:0.25 (w/w); IPM was used as the oil phase for all microemulsion systems

then analyzed using a confocal microscope (LEICA TCS-SP2 SE, Manheim, Germany) $)^{30,31}$. Fluorescence emission was detected using the spectral detector set in the 400-600 nm range. The detector settings were kept constant for all images.

\section{RESULTS AND DISCUSSION}

Characterisation of the microemulsion system: The construction of a pseudo-ternary phase diagram is essential to determine the range of concentrations and the ratios of components in the region in which the oil in water microemulsion exists. The pseudo-ternary phase diagram is shown in Fig. 1. The microemulsion formulation was composed of the nonionic surfactants Tween ${ }^{\circledR} 80$ and Span ${ }^{\circledR} 80$, PEG 400 as a cosurfactant and isopropyl myristate as an oil phase. Among the various weight ratios of Tween ${ }^{\circledR} 80$ plus Span ${ }^{\circledR} 80$ to PEG 400 that were investigated in the microemulsion systems, the ratio of 1:1 had the largest microemulsion area, indicating that the thermodynamic stability of the microemulsion is the greatest at this ratio. Tween ${ }^{\circledR} 80$ and Span ${ }^{\circledR} 80$ were chosen as surfactants for microemulsion. The mixture hydrophilelipophile balance (HLB) of Tween ${ }^{\circledR} 80$ and Span ${ }^{\circledR} 80$ was matched with isopropyl myristate (IPM). When it is similar to the required HLB of the microemulsion oil phase, the system provides the minimum energy condition for microemulsion formation. This method was in accordance with the rule of "the likes dissolves each other" since both of them had a long fatty acid structure which suggested that Tween $\circledast 80$, Span $® 80$ and isopropyl myristate had better compatibility, predicting a stable microemulsion can be obtained from them. In addition, Tween ${ }^{\circledR} 80$ could insert into the oil drop surface uniformly and form a homogeneous interface. Similar results were obtained for quercetin in $\mathrm{o} / \mathrm{w}$ microemulsion drug delivery system with Tween 80 and ethyl oleate ${ }^{3}$. In this test, the 1:1 ratio showed excellent solubility and exhibited a skin permeation-enhancing effect for ACADFE, which was used as a model of a sparingly soluble drug. The effect of the drug on the size, size distribution and zeta potential of the microemulsion droplets at $25 \% \mathrm{C}$ was investigated by dynamic light scattering (Figs. 2 and 3). According to the DLS results, the addition of ACADFE to the microemulsion increased the mean droplet diameter. The average droplet sizes of unloaded and ACADFEloaded microemulsions with surfactant-to-oil ratios of 70:30 were $21.7 \pm 2.04$ and $23.6 \pm 2.11 \mathrm{~nm}$ in diameter, respectively. After loading with ACADFE, the average droplet size increased to $23.6 \mathrm{~nm}$ (Fig. 2). Similar results were obtained for quercetin o/w microemulsion drug delivery system with Tween ${ }^{\circledR} 80$ and ethyl oleate ${ }^{3}$. Based on these studies, it can be concluded that this trend is the result of the following: The incorporation of quercetin in the microemulsion system increased the droplets size slightly in the systemdue to quercetin molecule should be mainly dissolved or dispersed into the emulsifying membrane layer (composed of surfactant and cosurfactant) with small amount of quercetin partitioning into oil phase, which leads to the change of microemulsion droplet size.

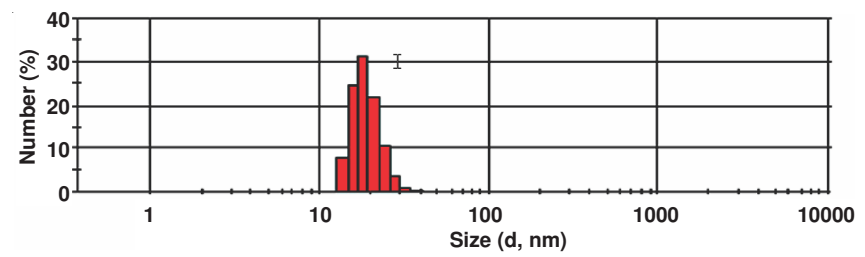

Fig. 2. Size distribution of ACADFE microemulsion (present work)

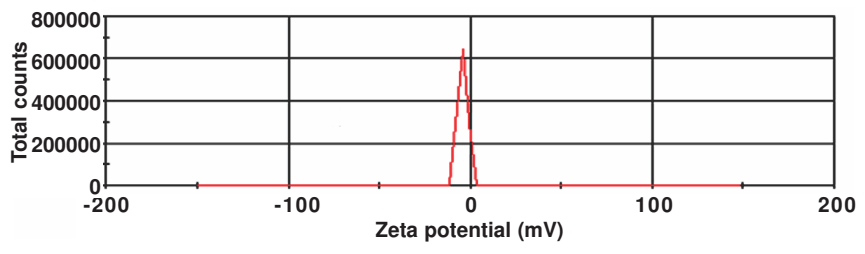

Fig. 3. Zeta potential of ACADFE microemulsion

Stability of the microemulsion system: The ACADFE contents of the microemulsions that were stable after 1,2 and 3 months were found to be $99.1,98.9$ and $98.3 \%$, respectively. The results reveal that the ACADFE-loaded microemulsion was stable during the study period.

Tissue distribution of quercetin in rats via the percutaneous route: The microemulsion formulation was used for the in vivo study. In the tissue distribution experiment, the method of HPLC was used. The chromatograms showed stable baselines, as well as displaying good resolution among quercetin in tissues. Figs. 4 and 5 showed that the microemulsion significantly enhanced $(p<0.05)$ the in vivo penetration of quercetin into the heart and kidneys relative to the penetration observed with the control formulation up to $12 \mathrm{~h}$ post-application. After this period, the concentrations of quercetin were stable in heart and kidneys, possibly due to the targeting of the heart and excretion by the kidneys. These results demonstrated the passive targeting properties of microemulsions 
towards heart and kidneys organs (Figs. 4 and 5). These results indicated that ACADFE-loaded microemulsions could successfully target the heart and correspondingly improved the efficacy of ACADFE to treat heart diseases. The reasons were mainly due to microemulsion encapsulating multiple molecules of drug to bind the receptors. Secondly, surfactants can speed the spread of droplets of drug and the disaggregation of microemulsion, so the drug on the heart surface can be absorbed rapidly.

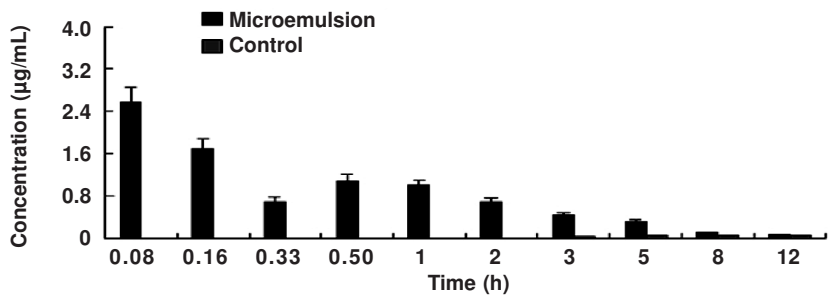

Fig. 4. Concentration-time profile of ACADFE in the heart

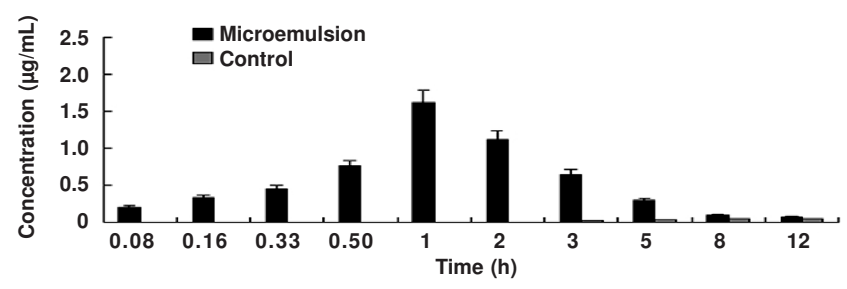

Fig. 5. Concentration-time profile of ACADFE in the kidney

in Vivo skin permeation mechanism: The penetration pathway of ACADFE within the skin samples was studied using confocal laser scanning microscopy. Representative fluorescence images of ACADFE in skin samples are shown in Fig. 6. First, the effect of ACADFE penetration into skin is discussed. At $10 \mathrm{~min}$, the green fluorescent signal of ACADFE was predominantly located around the follicle (Fig. 6a). The results of the present study indicated that the penetration of ACADFE proceeded via the follicular at the beginning of the penetration process and the lag time was shortened if it was applied in the microemulsion. Next, the pathway for ACADFE penetration into the skin is discussed. After $7 \mathrm{~h}$ of exposure of the skin to the o/w microemulsion, the fluorescent signal of ACADFE was predominantly located around the non-follicular region (Fig. 6b), indicating that the ACADFE penetration into skin proceeded via the transcellular pathway. Based on this result, it can be concluded that penetration proceeds via the transcellular route for the following reason: Some $\mathrm{S}$ and $\mathrm{CoS}$ in the microemulsion could be deposited at the interface with the skin, where they acted as enhancing agents. Consequently, permeation across transcellular lipids was the main pathway for penetration of the microemulsion. In particular, this route was found to be dominant for the microemulsion containing ACADFE.

The potential of a topical formulation to be used as a delivery system should be evaluated not only in terms of carrier capacity and percutaneous drug absorption but also in terms of its tolerability and toxicity.

\section{Conclusion}

The systemic delivery of ACADFE in an o/w microemulsion via the percutaneous route has been demonstrated. In addition,

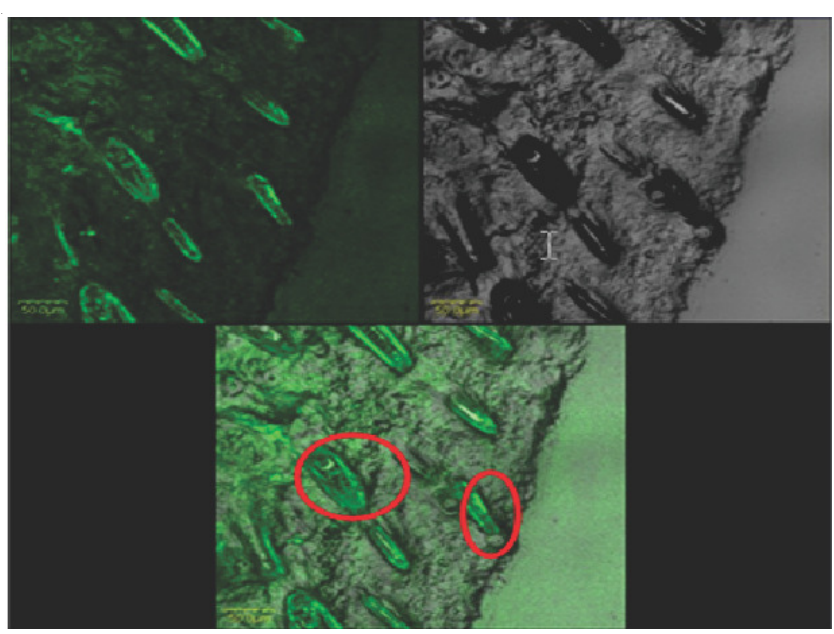

(a) $10 \min \times 300$

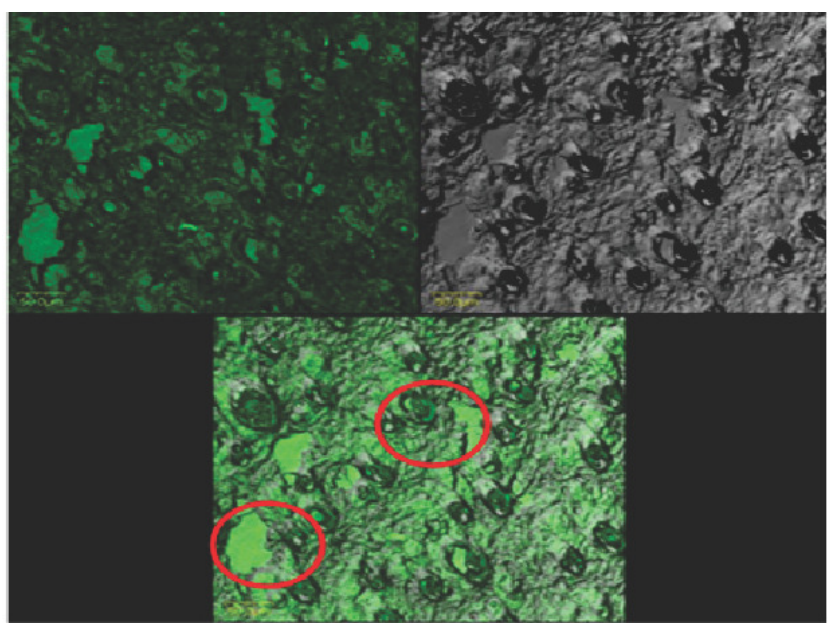

(b) $7 \mathrm{~h} \times 300$

Fig. 6. Fluorescence images of skin penetration after $10 \mathrm{~min}$ (a) and $7 \mathrm{~h} \mathrm{(b)}$

the microemulsion enabled the transdermal delivery and homogeneous biodistribution of the drug in the target tissue. These findings represent a unique contribution to the field of topical delivery of ACADFE drugs and demonstrate the potential advantages of using this vehicle in future preclinical and clinical studies to treat noncancerous and cancerous diseases.

\section{ACKNOWLEDGEMENTS}

This work was supported by the Ministry of Science and Technology of Jilin Province, P.R. China (Grant No. 20110919).

\section{REFERENCES}

1. X.H. Yang, Y.Y. Liu, L.J. Liu and X.P. Zhou, Acta Pharm. Sin., 35, 752 (2000).

2. P. Bansal, P. Paul, J. Mudgal, P.G. Nayak, S.T. Pannakal, K.I. Priyadarsini and M.K. Unnikrishnan, Exp. Toxicol. Pathol., 12, 1 (2011).

3. Y. Gao, Y.Q. Yang, Y.Q. Ma, A.H. Yu, F.Q. Cai, W. Shao and G.X. Zhai, Colloid. Surf. B, 71, 306 (2009).

4. B.H. Havsteen, Pharmacol. Ther, 96, 67 (2002).

5. S.W. Kim, C.E. Kim and M.H. Kim, Biochem. Biophys. Res. Commun., 415, 602 (2011).

6. R. Kleemann, L. Verschuren, M. Morrison, S. Zadelaar, M.J. van Erk, P.Y. Wielinga and T. Kooistra, Atherosclerosis, 218, 44 (2011).

7. A. Kumari, S.K. Yadav, Y. Pakade, B. Singh and S.C. Yadav, Colloid. Surf. B, 80, 184 (2010).

8. P.A. Omololu, J.B. Rocha and I.J. Kade, Exp. Toxicol. Pathol., 63, 249 (2010). 
9. M.M. Su, X.F. Yu, S.C. Qu, H.L. Xu and D.Y. Sui, Chin. Pharmacol. Bull., 23, 1593 (2007)

10. R. Gannu, C.R. Palem, V.V. Yamsani and S.K. Yamsani, Int. J. Pharm., 388, 231 (2010)

11. L.T. Hu, H.Y. Wu, F. Niu and C.H. Yan, Int. J. Pharm., 420, 251 (2011)

12. C. Jullian, L. Moyano, C. Yañez and C. Olea-Azar, Spectrochim. Acta A, 67, 230 (2007).

13. M.A. Bolzinger, S. Brianc, J. Pelletier, H. Fessi and Y. Chevalier, Eur. J. Pharmacokinet. Biopharm., 68, 446 (2008).

14. V.F. Lvovich, E. Matthews, A.T. Riga and L. Kaza, J. Control. Rel., 145, 134 (2010).

15. Y.H. Tsai, K.F. Lee, Y.B. Huang, C.T. Huang and P.C. Wu, Int. J. Pharm., 388, 257 (2010).

16. J.S. Yuan, M. Ansari, M. Samaan and E.J. Acosta, Int. J. Pharm., 249, 130 (2008).

17. J. Choi, M.K. Choi, S.J. Chung, C.K. Shim and D.D. Kim, Int. J. Pharm., 10, 1016 (2011)

18. R.M. Hathout, T.J. Woodman, S. Mansour and N.D. Mortada, Eur. J. Pharm. Sci., 40, 188 (2010).

19. W. Chaiyana, K. Saeio, W.E. Hennink and S. Okonogi, Int. J. Pharm., 401, 32 (2010)
20. G.M. Maghraby, Colloid. Surf. B, 75, 595 (2010).

21. A.C. Sintov, H.V. Levy and S. Botner, J. Control. Rel., 148, 168 (2010).

22. A.H. Yu, C.Y. Guo, Y.B. Zhou, F.L. Cao and W.W. Zhu, Int. Immunopharmacol., 10, 1305 (2010).

23. F.C. Rossetti, L.B. Lopes, A.R. Carollo and J.C. Thomazini, J. Control. Rel., 155, 400 (2011)

24. X.R. Li, Y.T. Yue, Y.X. Zhou, Y.T. Fan and T. Fan, Int. J. Pharm., 407, 158 (2011)

25. L. Montenegro, C. Carbone and G. Puglisi, Int. J. Pharm., 405, 162 (2011).

26. F.T. Vicentini, T.R. Simi, J.D. Ciampo, N.O. Wolga, D.L. Pitol, M.M. Iyomasa, M.L. Bentley and M.J. Fonseca, Eur. J.Pharmacokinet. Biopharm., 69, 948 (2008).

27. W.W. Zhu, C.Y. Guo, A.H. Yu, Y. Gao, F.L. Cao and G.X. Zhai, Int. J. Pharm., 378, 152 (2009).

28. L. Gan, Y. Gan, C.L. Zhu, X.X. Zhang and J.B. Zhu, Int. J. Pharm., 365, 143 (2008).

29. Q.Z. Zhang, X.G. Jiang, W.M. Jiang, W. Lu, L.N. Su and Z.Q. Shi, Int. J. Pharm., 275, 85 (2004).

30. L.M.P.C. Araújo, J.A. Thomazine and R.F.A. Lopez, Eur. J. Pharmacokinet. Biopharm., 75, 4 (2010).

31. M. Changez, J. Chander and A.K. Dinda, Colloid. Surf. B, 48, 58 (2006). 